\title{
Synthèse
}

Génétique et génomique

\section{La génétique du bar (Dicentrarchus labrax L.)}

\author{
Béatrice Chatain ${ }^{1}$ \\ Hervé Chavanne ${ }^{2}$ \\ 1 Ifremer \\ Station expérimentale d'aquaculture \\ Chemin de Maguelone \\ 34250 Palavas-les-Flots \\ France \\ <beatrice.chatain@ifremer.fr> \\ 2 Istituto sperimentale italiano Lazzaro \\ Spallanzani \\ Via Le Forlanini, 23 \\ 20100 Milan \\ Italie \\ <panamelio@hotmail.com>
}

\begin{abstract}
Résumé
La production de bar, espèce majeure de la pisciculture méditerranéenne, repose encore, dans bien des cas, sur des animaux issus de parents sauvages. Pourtant, l'amélioration génétique est une des clés du développement de toutes les filières d'élevage optimisées. Trois types d'amélioration peuvent être réalisés lorsqu'il s'agit de poissons : i) la production de populations monosexes. Pour le bar, ce serait du monosexe femelle, car les femelles sont plus grosses que les mâles (gain de croissance potentielle de 20$30 \%$ ). Cependant, le déterminisme du sexe chez cette espèce est très complexe, car il fait intervenir des composantes génétiques et environnementales. Le monosexage femelle n'est donc pas encore maîtrisé en routine, même si on peut parfois obtenir des lots presque entièrement femelles; ii) les manipulations chromosomiques, au premier rang desquelles l'induction de triploïdes qui aboutit à des animaux viables mais stériles. Cela devrait permettre d'éviter les ralentissements de croissance pour les poissons de plus de $600 \mathrm{~g}$ et permettre la protection du progrès génétique. L’induction triploïde est maîtrisée chez le bar, mais n'est pas utilisée, car elle provoque des retards de croissance; iii) la sélection, qui montre sur la plupart des caractères testés des possibilités de gains importants, probablement du fait que l'espèce n'est actuellement pas encore domestiquée. La "boîte à outils " de l'amélioration génétique est donc disponible chez le bar et les premiers programmes de sélection réellement optimisés sont aujourd'hui en cours de développement.
\end{abstract}

Mots clés : amélioration génétique ; Dicentrarchus labrax ; génétique des populations ; pisciculture ; poisson de mer ; zone méditerranéenne.

Thèmes : productions animales ; pêche et aquaculture ; amélioration génétique.

\section{Abstract \\ Genetics of European seabass (Dicentrarchus labrax L.)}

The production of sea bass, a major species in Mediterranean fish farming, still relies in many cases on the production of fingerlings issued from wild breeders. Still, genetic improvement is a major key to sustainable development for all optimized forms of animal production. Three types of genetic improvement can be acheived in fish: i) the production of monosex populations. For seabass, monosex female populations would be the objective as females grow $20-30 \%$ faster than males. However, sex determination in this species is complex, impacted by both genetic and environmental factors. Hence, production of monosex populations is not yet a routine operation, although in some cases almost 100\% female batches have been obtained; ii) chromosome set manipulations, the most important being triploid induction, which produces viable but sterile fish. The aim in seabass would be to increase late growth and protect genetic gain. Although triploid induction is mastered in seabass, it is not used as it induces some growth retardation; iii) selective breeding, which promises important productivity gains on almost all tested traits, probably due to the fact that the species is not yet domesticated. The genetic improvement "toolbox" is therefore available in European sea bass, but truly optimised commercial breeding schemes have only recently started.

Key words: breeding; Dicentrarchus labrax; fish culture; Mediterranean zone; population genetics; sea fishes.

Subjects: animal productions; fishing and aquaculture; genetic improvement. 


\section{Historique de la filière et de la domestication de l'espèce}

Les premiers élevages de bar débutent dans les années 1970 et, comme pour presque toutes les espèces marines, sont réalisés à partir de juvéniles collectés dans le milieu naturel. Ces juvéniles (figure 1) sont élevés en extensif dans des lagunes semi-artificielles du type "valliculture" italienne.

Dans les années 1980, afin de faire face à une compétition grandissante entre grossisseurs pour l'accès aux alevins sauvages et à la diminution des ressources naturelles, apparaissent en France et en Italie, les premières unités d'élevage intensif de petite taille qui permettent de produire une source alternative de juvéniles. La maîtrise complète du cycle est acquise, et les techniques de reproduction et d'alevinage permettent d'atteindre régulièrement des taux de survie compatibles avec le développement d'une véritable industrie. La diffusion de ce savoir-faire permet progressivement d'accroître les volumes de production dans toute l'Europe, production qui était essentiellement basée sur des stocks de géniteurs sauvages jusque dans les années 1990.

À partir de là, les premiers programmes de sélection commencent à se développer sérieusement en France, puis quelques tentatives timides sont réalisées en Grèce, en Espagne et en Italie. Aujourd'hui, la plupart des juvéniles de bar produits en Europe (350 millions en 2006 ; FEAP, 2007) sont encore issus de géniteurs sauvages ou d'animaux de première génération, moins de $20 \%$ étant issus de géniteurs sélectionnés de deuxième ou troisième génération.

Le système d'élevage qui domine aujourd'hui, pour le grossissement, est la cage

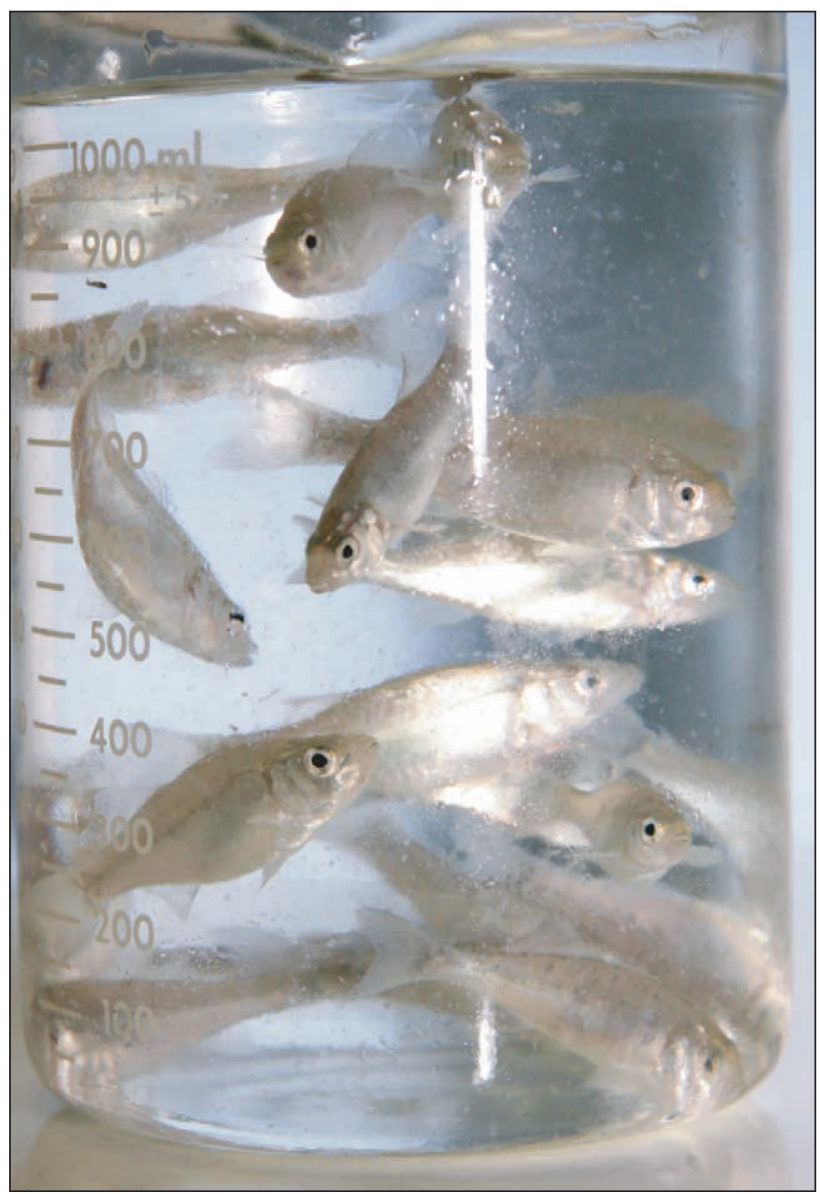

Figure 1. Juvéniles de bar (trois mois, un gramme), Dicentrarchus labrax.

Figure 1. Juvenil bass (3 months, 1 gram), Dicentrarchus labrax.

immergée en mer. La durée d'élevage, jusqu'au poids commercial, est extrêmement variable, car dépendante de la température des sites de grossissement qui se répartissent depuis la côte Atlantique, jusqu'à la Méditerranée, voire jusqu'en mer Rouge. Les différences moyennes annuelles entre les extrêmes sont de 5-6 ${ }^{\circ} \mathrm{C}$. Près de la moitié du volume commercial (82 000 tonnes en 2005: FEAP, 2007) est constituée par le bar portion de 300/400 g, $27 \%$ par celui de 400/600 g et $17 \%$ par celui de 200/300 g. Les animaux ayant dépassé le kilogramme ne représentent qu'un marché de niche $(1 \%$ de la production). Ces proportions varient selon les pays : l'Espagne, par exemple, ne produit quasiment pas de bars de moins de $300 \mathrm{~g}$ et la Turquie de plus de 800 g. En France, 20 \% de la production consiste en des poissons de plus de $800 \mathrm{~g}$.

\section{Structure génétique des populations naturelles et des populations d'élevage}

Les populations naturelles de bars ont été particulièrement bien étudiées. De nombreux travaux, faisant appel aux marqueurs moléculaires, ont permis de diviser l'aire de répartition de l'espèce en trois grands groupes : Nord Atlantique, Ouest Méditerranéen et Est Méditerranéen (Patarnello et al., 1993; Naciri et al., 1999). Deux zones de contacts ont été identifiées sur le front océanique d'Almeria-Oran séparant les groupes Atlantique et Ouest Méditerranéen et au niveau du haut-fond Siculo-Tunisien séparant les groupes Ouest et Est Méditerranéens. Les groupes Atlantique et Ouest Méditerranéen sont relativement homogènes, même si de récentes études indiquent une structure probablement plus complexe des populations localisées autour des îles anglaises (Fritsch et al., 2007). Le groupe Est Méditerranéen, plus hétérogène, est structuré en souspopulations qui reflètent la présence de bassins régionaux. Des différences entre populations de mer et de lagune existent aussi si l'on se réfère aux études réalisées 
avec des marqueurs allozymes (Allegrucci et al., 1997 ; Lemaire et al., 2000). L'analyse de la distribution des marqueurs nucléaires entre populations démontre que, parmi 13 allozymes non neutres, six loci marquent la différence entre les échantillons de mer ou de lagunes, ce qui suggère une divergence génétique entre habitats.

A contrario, les populations d'élevage, non soumises à la sélection, n'ont été que très peu étudiées. Les quelques études qui ont été menées à l'aide de marqueurs moléculaires, ont révélé, parfois, des réductions significatives de variabilité, mais, le plus fréquemment, un niveau de variabilité en concordance avec l'équilibre de Hardy-Weinberg (témoin d'un équilibre de la fréquence des allèles et des génotypes au cours des générations), ce qui indique que ces stocks sont encore largement variables et constitués (ou régulièrement renouvelés), avec des géniteurs sauvages (Patarnello et al., 1993; Bahri-Sfar et al., 2005 ; Sola et al., 1998).

\section{Interactions}

\section{et différences}

entre les populations sauvages et d'élevage

L'introduction de populations non autochtones dans les populations naturelles est courante chez le bar, en raison des échanges commerciaux de juvéniles qui s'effectuent à travers toute l'Europe et des animaux qui s'échappent régulièrement des cages flottantes; il n'existe pas de législation sur le contrôle des échappés pour cette espèce. Ce phénomène pourrait s'aggraver avec les approvisionnements de géniteurs connus pour avoir de meilleures performances d'élevage (Gorshkov et al., 2004). Cependant, un seul cas avéré de "contamination" des populations de Méditerranée Est par une population de Méditerranée Ouest a réellement été identifié (Bahri-Sfar et al., 2005).

Très peu d'expériences de testage ont été conduites, à ce jour, sur cette espèce. Il n'existe qu'une seule étude comparant une population domestiquée et une population sauvage dans un environnement tropical en mer Rouge, en Israël, qui rapporte des différences significatives pour de nombreux caractères d'intérêt économique comme la survie, la croissance, la composition corporelle (Gorshkov et al., 2004). Les travaux de Vandeputte et al. (2007), quant à eux, prévoient des modifications profondes du déterminisme du sexe chez les populations sauvages au cours de la domestication ou lorsqu'elles sont sélectionnées pour la croissance. Davantage d'informations sont attendues d'un projet européen (Competus [COOP-CT-2005-017633]. Genetic improvement of farmed seabass, Dicentrarchus labrax: strain testing and response to selection) qui a trouvé son achèvement en 2008, sur les performances comparées de cinq populations sauvages issues d'origines géographiques couvrant toute l'aire de répartition de l'espèce.

\section{Amélioration}

\section{par le contrôle du sexe}

La production de populations monosexes est une pratique relativement courante, dans les filières poissons (truites et tilapias en particulier), qui permet de ne produire que des individus d'un seul sexe, celui qui présente les caractères les plus avantageux pour la production. Pour le bar, espèce gonochorique, c'est le sexe femelle qui est le plus intéressant, car il présente un avantage de croissance non négligeable. Le dimorphisme pondéral, important dans les jeunes stades (70\%), diminue et se stabilise autour de 20-30\% chez le poisson de deux ans et plus (Blazquez et al., 1999 ; Pavlidis et al., 2000 ; Saillant et al., 2001b; Saillant et al., 2003 ; Gorshkov et al., 2004).

La production de populations monoparentales nécessite de connaître le déterminisme du sexe de l'espèce. Ce déterminisme, chez le bar, a fait l'objet de nombreux travaux (voir revue de Piferrer et al., 2005), parce que le sex-ratio, chez cette espèce, est fortement déséquilibré dans les conditions d'élevage où 70 à $99 \%$ des alevins produits sont des mâles. Toutes ces études mènent à la conclusion que le sexe, chez le bar, est déterminé à la fois par des facteurs génétiques et environnementaux, le principal étant la température.

Côté génétique, un déterminisme du sexe simple (du type XY ou ZW) a été exclu par des études impliquant l'utilisation de géniteurs inversés sexuellement ou la gynogenèse ( $c f$. "Amélioration par le contrôle de la maturation "), et Vandeputte et al. (2007) avancent l'hypothèse de l'existence d'une composante polygénique. La part génétique est essentiellement additive, comme le démontrent également Saillant et al. (2002), liée à la croissance, et est du même ordre de grandeur que la part sous contrôle environnemental.

Côté environnemental, une exposition à des basses $\left(13^{\circ} \mathrm{C}\right)$ ou hautes $\left(20^{\circ} \mathrm{C}\right)$ températures, pendant toute la période de différenciation du sexe (de l'éclosion jusqu'à ce que l'animal atteigne 160$170 \mathrm{~mm}$ ), a des effets masculinisants. À l'inverse, une augmentation de température (de 15 à $20^{\circ} \mathrm{C}$, par exemple) entre 18 et $60 \mathrm{~mm}$ permet un accroissement significatif de la proportion de femelles dans la descendance. Malgré toutes ces études, aucune population $100 \%$ femelle n'a jamais été obtenue et un protocole de routine impliquant des variations de température précises permettant de dévier systématiquement et fortement le sexratio vers le sexe femelle manque encore à ce jour.

La production de populations monosexes peut uniquement être obtenue avec des traitements hormonaux exogènes qui conduisent à une réversion complète (Blazquez et al., 1998; Chatain et al., 1999), mais cette pratique est interdite pour des animaux destinés à la consommation en Europe.

\section{Amélioration}

\section{par le contrôle de la maturation}

La stérilisation en amélioration génétique est principalement utilisée pour protéger le progrès génétique et permettre la vente d'un cheptel amélioré sans qu'il soit possible à l'acheteur de le reproduire. Elle est aussi employée pour éviter le ralentissement de croissance dû, chez la majorité des espèces, à la dépense énergétique occasionnée par la gamétogenèse. Chez le bar, cette dernière est plus précoce chez le mâle que chez la femelle (Bruslé et Robin, 1984) comme chez de nombreux poissons. Elle concerne un tiers des mâles à l'âge d'environ deux ans $(200 \mathrm{~g}), 100 \%$ à l'âge de trois ans ayant 
atteint la taille commerciale la plus fréquente de $400 \mathrm{~g}$ et toutes les femelles d'environ quatre ans (600 g).

Pour les poissons, dont les gonades sont inaccessibles à la castration physique, la meilleure méthode de stérilisation est la triplöidisation (production d'individus possédant trois jeux de chromosomes, deux provenant de la mère et un du père). Elle peut être obtenue de deux façons :

- soit en traitant les œufs issus d'un croisement de deux individus diploïdes (2n) pour empêcher l'expulsion du second globule polaire (second jeu de chromosomes d'origine maternelle) au cours de la méiose ;

- soit par un croisement entre individus tétraplö̈des (4n) et diploïdes. Les tétraplö̈des sont obtenus par un traitement des oufs au moment de la première mitose pour bloquer la cytodiérèse aboutissant à la première division cellulaire.

Les premiers essais de triploïdisation ont été réalisés, chez le bar, à la fin des années 1990 (Colombo et al., 1995 ; Felip et al., 1997) et des traitements optimaux (par la pression ou par le froid) permettent aujourd'hui la production de $100 \%$ de triploïdes (Peruzzi et Chatain, 2000). Les œufs doivent être traités, 2 min après la fécondation, à une pression de 800 psi ou immergés pendant $20 \mathrm{~min}$ dans de l'eau de mer à $0{ }^{\circ} \mathrm{C}$. La stérilité fonctionnelle caractérise les deux sexes sous forme triploïde, mais un développement partiel des testicules est observé chez le mâle (40-50 \% de la taille d'un testicule normal). Les performances de croissance des triploïdes sont malheureusement comparables (parfois), mais plus généralement, inférieures de $20 \%$ à celles des témoins diploïdes. Certains caractères de qualité sont, cependant, améliorés, e.g. le rendement de carcasse et le facteur de condition sont supérieurs, et le rapport gonadosomatique très inférieur (Felip et al., 1999 ; Peruzzi et al., 2004).

Les seuls tétraploïdes de bar qui aient jamais été produits (par traitement pression) n'ont pas survécu plus de deux mois (Libertini et al., 2002 ; Peruzzi et Chatain, 2003).

Des traitements similaires à ceux qui sont pratiqués pour la triploïdie, mais appliqués à des œufs fécondés avec du sperme irradié (dont le matériel génétique est donc détruit), ont permis de produire des lignées gynogénétiques. Ces animaux méiogynogènes, se développant à partir du seul matériel génétique maternel, ne sont pas produits pour la stérilité, mais peuvent être utilisés pour fixer certains caractères qui ne seraient portés que par les femelles ou encore comme base pour produire des clones. Ils ont une survie d'environ $50 \%$ de celle des témoins, grossissent de façon similaire et ont un potentiel reproductif comparable à celui des diploïdes i.e. qualité d'œufs et de sperme identiques en termes de volume, de qualité et de capacité de fécondation (Peruzzi et al., 2004 ; Francescon et al., 2005).

\section{Amélioration par la sélection}

La sélection du bar est très récente, et les programmes de sélection menés par les industriels n'amorcent, aujourd'hui, au mieux, que leur quatrième génération. Les travaux scientifiques, menés dans ce domaine, démontrent tous que le bar est une espèce de choix pour la sélection, avec la possibilité d'obtenir, au moins dans les premières générations, des gains importants sur la croissance, la morphologie et la qualité de la chair sans nécessairement recourir à des méthodes lourdes de sélection familiale. Ce potentiel repose principalement sur l'existence, d'une part, d'héritabilités fortes pour la plupart des caractères d'intérêt économique et, d'autre part, de corrélations génétiques favorables entre les différents caractères d'intérêt.

On peut ainsi envisager d'améliorer le poids à taille commerciale (400 g) de 25 à $30 \%$ par génération, par sélection des $5 \%$ d'animaux les plus lourds, car l'héritabilité $\left(h^{2}\right)$ du poids est comprise entre $0,52 \pm 0,07$ et $0,64 \pm 0,07$; des valeurs similaires sont rapportées pour la longueur (Dupont-Nivet et al., 2008 ; Saillant et al., 2006). Cette sélection devrait peu modifier la forme des animaux et augmenter légèrement la proportion de gras musculaire. Ces deux derniers caractères étant également bien héritables $\left(\mathrm{h}^{2}=0,39-0,54\right.$ pour le coefficient de condition; $h^{2}=0,48-0,68$ pour le gras musculaire), ils peuvent faire l'objet d'une sélection avec un potentiel de réponse conséquent, ce qui permettrait de proposer des animaux plus allongés et/ou moins gras, préférés par les consommateurs (Dupont-Nivet et al., 2008 ; Chatain et al., 2007). Une forte corrélation entre les valeurs génétiques des caractères de croissance à différents âges (moyenne entre les âges : 0,70) suggère que les valeurs génétiques sont stables dans le créneau d'âge étudié et que, par conséquent, la sélection de ces caractères pourrait être réalisée à des stades relativement précoces (ici dès $90 \mathrm{~g}$ : Saillant et al., 2006).

Une autre corrélation intéressante et forte $(r=0,50 \pm 0,09)$ existe entre le sexe $\left(\mathrm{h}^{2}=0,62 \pm 0,12\right)$ et la croissance qui laisse présager une augmentation de la proportion de femelles par une simple sélection sur le poids. Une fois corrigée pour le dimorphisme sexuel, cette relation positive est maintenue, ce qui renforce l'idée que des gènes communs participent à la croissance et au déterminisme du sexe. Elle laisse entrevoir une évolution du sex-ratio dans les fermes au fur et à mesure des générations de sélection ou de domestication. La simple domestication devrait amener à un équilibre mâlefemelle au bout de sept à huit générations et une sélection sur la croissance devrait déplacer cet équilibre vers des populations comprenant plus de femelles que de mâles (Vandeputte et al., 2007).

D'autres gains importants sont attendus sur les rendements de transformation, objectif intéressant pour l'avenir, même si le bar est actuellement vendu entier. Pour un rendement de carcasse moyen de 83 à $87 \%$, l'héritabilité varie de 0,54 à 0,74 selon les sites, autorisant un gain de rendement de 1,8 à $3 \%$ par génération avec une pression de sélection modérée (20\%). De même, le rendement en filet ( $48 \%$ en filet pelé) pourrait être amélioré de $2 \%$ par génération avec la même pression de sélection (Haffray et al., 2006 ; Chatain et al., 2007). Cependant, en l'absence de prédicteurs externes fiables, ce type de sélection implique l'abattage de collatéraux en nombre important, avec identification des généalogies, ce qui peut rendre ces critères difficiles à mettre en application.

Dans les années proches, des données seront disponibles (Aquafirst (SSP8CT-513692). Combined genetic and functional genomic approaches for stress and disease resistance marker assisted selection in fish and shellfish. http://aquafirst.vitamib.com/) sur les gènes impliqués dans la régulation de la réponse immunitaire responsable de la résistance aux pathogènes (Chistiakov et al., 2007). Deux types d'effets peuvent perturber l'efficacité des programmes de sélection, les effets maternels et les interactions génotype-environnement. 
Les premiers ne poseront apparemment pas de problèmes particuliers chez le bar, car ils sont de courte durée. En effet, les effets parentaux qui ont été identifiés, dès le plus jeune âge (11 jours), sur la croissance, la survie et le facteur de condition s'estompent progressivement pour disparaître totalement à l'âge de quatre mois (Dupont-Nivet et al., 2008 ; Garcia De Leon et al., 1998 ; Saillant et al., 2001a). Cet effet maternel de courte durée pourrait s'expliquer par la petite taille des oufs de bar (environ $1 \mathrm{~mm}$ de diamètre) qui n'influencerait pas durablement le développement larvaire. Les interactions génotype-environnement pour la croissance sont importantes entre des environnements extrêmes (corrélations génétiques variant de $0,01 \pm 0,26$ à 0,51 \pm 0,19; Saillant et al., 2006), mais faibles quand on compare des sites industriels (corrélations génétiques variant de 0,75 $\pm 0,06$ à 0,98 \pm 0,02; Dupont-Nivet et al., 2008). Pour le sex-ratio, les variations observées chez des familles élevées sous des profils de température différents prouvent, pour les deux sexes, l'existence d'une interaction génotype-environnement dans le déterminisme du sexe qui pourrait signifier l'existence de génotypes à sensibilité différentielle à la température (Saillant et al., 2002). Si tel est le cas, on devrait constater une élimination progressive de l'effet environnemental sur le déterminisme du sexe chez cette espèce, quand elle est soumise à une sélection de parents non sensibles aux effets des températures masculinisantes. De faibles interactions ont aussi été estimées pour des caractères de qualité testés dans des conditions d'élevage impliquant plusieurs sites d'élevage industriels (Haffray et al., 2006).

\section{Outils de l'amélioration génétique}

Les principaux outils qui ont été développés ces dernières années pour permettre le développement de l'amélioration génétique du bar sont :

- les marqueurs permettant de caractériser la variabilité génétique des populations et d'identifier les parents d'un individu ;

- les marqueurs physiques individuels ;

- la fécondation artificielle ;

- la cryoconservation.
L'analyse de la structure génétique des populations sauvages et d'élevage a, tout d'abord, reposé sur l'analyse de l'ADN mitochondrial et des variations d'allozymes (Patarnello et al., 1993 ; Allegrucci et al., 1997 ; Castilho et McAndrew, 1998). Ces marqueurs ont été progressivement supplantés par des marqueurs microsatellites (Garcia De Leon et al., 1995; Garcia De Leon et al., 1997 ; Bahri-Sfar et al., 2000 ; Bahri-Sfar et al., 2005 ; Castilho et Ciftci, 2005). Aujourd'hui, avec l'accroissement constant et rapide des nouvelles techniques en génomique, un grand nombre d'autres nouveaux marqueurs est en cours de développement chez cette espèce comme chez d'autres (Volckaert et al., 2006).

Les marqueurs microsatellites sont aussi utilisés en sélection pour l'assignation de parenté qui a permis d'utiliser, entre autres, des protocoles d'élevage en environnement commun (Dupont-Nivet et al., 2008 ; Garcia De Leon et al., 1998 ; Saillant et al., 2002 ; Saillant et al., 2006 ; Vandeputte et al., 2007). À côté des microsatellites, le nombre important, la variabilité, la stabilité et le nombre limité d'erreurs associées aux analyses réalisées avec les marqueurs SNP (Single Nucleotide Polymorphism), en font aussi de bons candidats pour ce type d'usage, mais il faudra que leur coût diminue sensiblement avant que leur diffusion ne s'élargisse. Le criblage de nouveaux SNP, dans de grandes banques EST (Expressed Sequence Tags), est en cours (Volckaert et al., 2006).

Le suivi généalogique ne peut cependant se faire que si les poissons sont physiquement et individuellement identifiés. Dans la pratique, en effet, l'analyse de l'ADN s'effectue a posteriori, à partir d'un mor- ceau de nageoire (figure 2B), prélevé sur chaque poisson qui reçoit, à cette occasion, une marque personnalisée avant d'être mélangé avec le reste de la population. Ce marquage physique peut être réalisé avec l'injection de marqueurs fluorescents (élastomères) sous la peau sur des poissons anesthésiés d'une longueur minimale de $50 \mathrm{~mm}$. Ils ne sont cependant pas utilisables sur de grandes populations car le nombre de couleurs et d'emplacements du marquage est limité ; de plus, leur temps de rétention n'est que de deux à trois mois. Les transpondeurs intégrés (PIT-Tag ; figure 2A), quant à eux, ne présentent aucune de ces limites, ils peuvent être utilisés sur des individus d'un poids d'environ $10 \mathrm{~g}$ en pratiquant une incision de la cavité abdominale ou, passés 30 g, injectés directement dans le muscle dorsal.

La fécondation artificielle du bar est peu pratiquée en production, car cette espèce pond naturellement dans les bassins. Cependant, pour effectuer des plans de croisements spécifiques ou pour pratiquer la polyploïdisation ou la gynogenèse, la fiabilisation de cette méthode s'est avérée indispensable. Le bar est un pondeur fractionnaire (trois à quatre pontes sur une saison de reproduction) qui mature sous le contrôle de la température et de la photopériode. Les œufs fécondés ont une taille d'environ 1,2 à 1,4 mm et la femelle pond environ 200000 œufs par kilogramme de biomasse (figure $3 \mathrm{~A}$ ).

Différents produits, comme les hormones antagonistes de GnRH ou de LHRH (gonadolibérines), permettent la synchronisation des pontes trois jours après le traitement ou l'allongement de la période de production de sperme chez les mâles et la production de pontes multiples chez

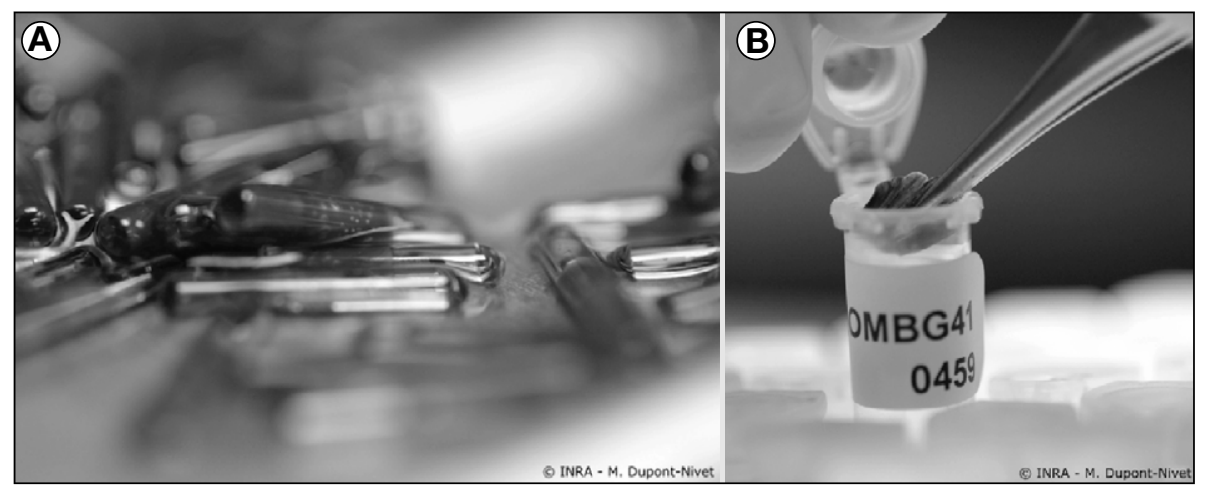

Figure 2. Marques électroniques individuelles PIT-Tag (A) et stockage de nageoires (B) pour analyses de parenté.

Figure 2. (A) PIT-Tag individual electronic tags; (B) fin stocking for genetic analyses. 


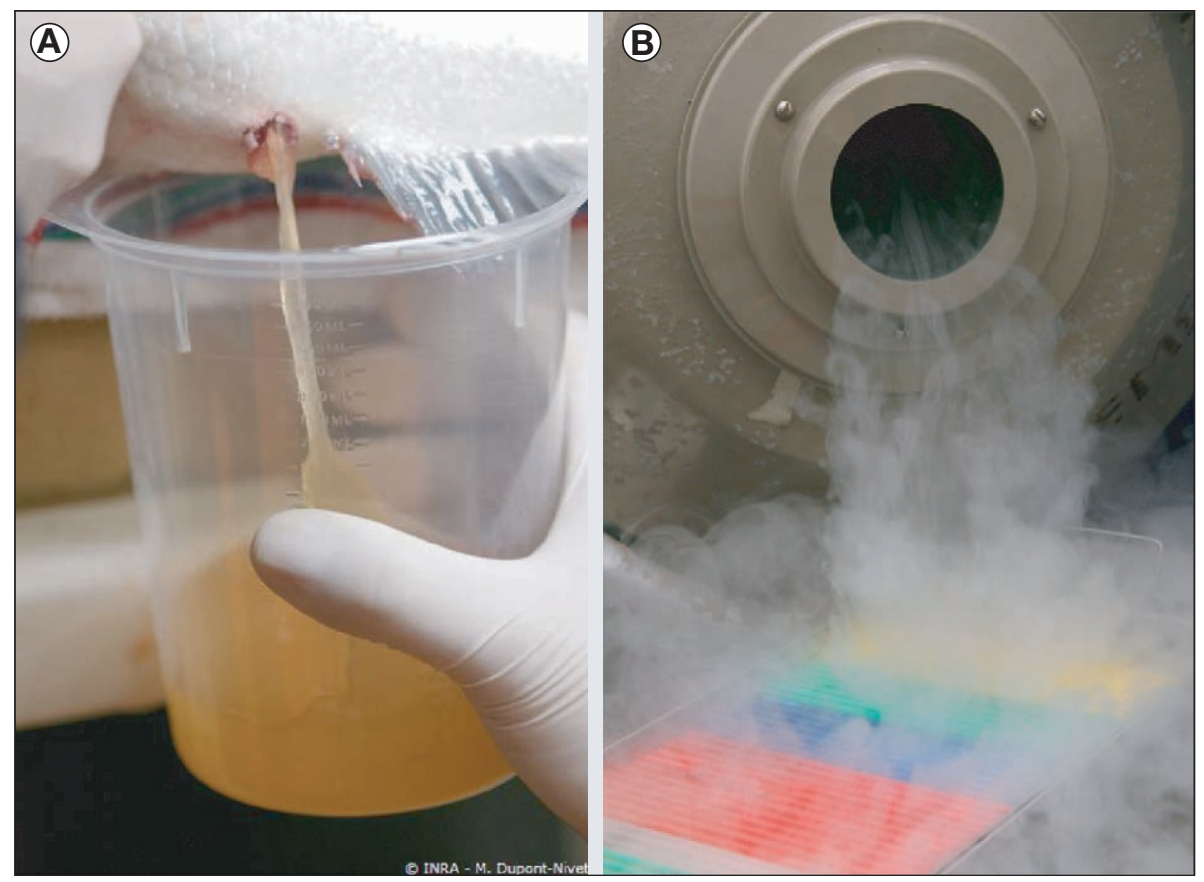

Figure 3. Récupération d'ovocytes de bar par massage abdominal (A) et paillettes de sperme dans I'azote liquide (B).

Figure 3. (A) Bass oocyte recuperation by abdominal massage; (B) sperm straws in liquid nitrogen.

la femelle (Fornies et al., 2001). Les deux limites majeures à la réalisation de grands plans de croisement pour l'espèce restent la surmaturation très rapide des oufs (moins de trois heures), une fois l'ovulation réalisée, et la difficulté à synchroniser à l'heure près un grand nombre de femelles. De tels plans sont cependant pratiqués de façon courante dans les organismes de recherche en France (Saillant et al., 2006 ; Vandeputte et al., 2007).

Cette pratique est facilitée par l'emploi de sperme congelé (figure 3B), méthode qui permet aussi de sécuriser les programmes de sélection en autorisant la conservation de plusieurs générations et la constitution de sauvegardes des noyaux de sélection. Différents protocoles de congélation sont aujourd'hui disponibles et efficaces pour conserver le sperme de bar (Fauvel et al., 1998 ; Sansone et al., 2002). Ils diffèrent par la qualité du cryoprotectant (diméthylsulfoxyde ou éthylène-glycol) et par la cinétique de baisse en température du sperme pendant le processus de cryoconservation. Un produit commercial donne aussi de bons résultats pour le bar (CryoFish - licence INRA-IMV-SYSAAF). Des essais ont été faits pour constituer des banques de spermes d'animaux sauvages, domestiques et sélectionnés avec des succès variables. Des cryobanques de taille respectable existent cependant aujourd'hui dans des organismes de recherche.

\section{Quel avenir pour l'amélioration génétique du bar?}

Actuellement, la majorité des bars produits en Europe est encore issue de géniteurs sauvages et les avancées qui ont été décrites ici, sur les méthodes d'amélioration génétique, ne sont que peu exploitées. Cet état de fait est une exception dans le domaine des productions animales ou végétales, secteurs dont l'économie dépend largement de l'amélioration génétique. La jeunesse de la pisciculture marine (une trentaine d'années) en est sans doute la cause, les espèces marines étant tout juste domestiquées voire en voie de domestication.

Les quelques pionniers qui ont investi dans le progrès génétique des espèces strictement marines (bar, daurade, turbot) sont des fermes grecques, espagnoles et françaises, ces derniers détenant aujourd'hui les générations de cheptels les plus avancées. La place prépondérante que tient l'industrie française dans ce domaine incombe, pour une grande part, à l'existence d'une interface recherche-industrie dans le domaine de l'amélioration génétique particulièrement efficace et unique en Europe : le Sysaaf (Syndicat des sélectionneurs avicole et aquacole français).

Parmi toutes les méthodes et outils d'amélioration utilisables pour le bar, la sélection est la plus employée, les caractères sélectionnés étant, pour l'instant, la croissance, la teneur en lipide du muscle et parfois la morphologie. Les méthodes employées sont la sélection familiale ou massale, l'efficacité de cette dernière ayant été largement améliorée grâce à l'emploi combiné de plusieurs outils permettant d'éviter la consanguinité, i.e. :

- la reproduction artificielle qui permet d'effectuer des croisements dirigés (géniteurs choisis pour maximiser la variabilité génétique);

- la cryoconservation qui permet d'effectuer des grands plans de croisement ;

- l'assignation de parenté par marqueurs microsatellites qui permet de contrôler les généalogies.

Les autres améliorations envisageables par la production de populations monosexes femelles et/ou la stérilisation par triploïdisation ne sont pas employées aujourd'hui par les industriels, la première n'étant pas encore maittrisée et la seconde parce qu'elle induit des pertes de croissance. Des recherches sont donc encore à mener dans ce secteur pour comprendre le déterminisme du sexe et la physiologie de l'animal triploïde.

Les prochaines avancées aujourd'hui attendues dans l'amélioration génétique du bar sont la description des performances en élevage de la ressource naturelle de l'espèce (populations sauvages) pour que les fermes puissent constituer leurs cheptels de géniteurs en pêchant les animaux qui ont les caractéristiques les plus avantageuses et la variabilité génétique la plus large.

\section{Références}

Allegrucci G, Fortunato C, Sbordoni V. Genetic structure and allozyme variation of seabass (Dicentrarchus labrax and D. punctatus) in the Mediterranean Sea. Mar Biol 1997; 128: 347-58.

Bahri-Sfar L, Lemaire C, Ben Hassine OK, Bonhomme F. Fragmentation of seabass populations in the western and eastern Mediterranean as revealed by microsatellite polymorphism. Proc R Soc Lond B Biol Sci 2000; 267 : 929-35. 
Bahri-Sfar L, Lemaire C, Chatain B, Divanach P, Ben Hassine OK, Bonhomme F. Impact de I'élevage sur la structure génétique des populations méditerranéennes de Dicentrarchus labrax. Aquat Living Resour 2005 ; 18 : 71-6.

Blazquez M, Carrillo M, Zanuy S, Piferrer F. Sex-ratios in offspring of sex-reversed seabass and the relationship between growth and phenotypic sex differentiation. J Fish Biol 1999: $55: 916-30$.

Blazquez M, Zanuy S, Carillo M, Piferrer F. Effects of rearing temperature on sex differentiation in the European seabass (Dicentrarchus labrax L.). J Exp Zool 1998; 281 : 207-16.

Bruslé J, Robin J. Sexualité du loup Dicentrarchus labrax en condition d'élevage controlé. In: Barnabé B, Billard R, eds. L'aquaculture du bar et des sparidés. Paris: Inra éditions, 1984.

Castilho R, Ciftci Y. Genetic differentiation between close eastern Mediterranean Dicentrarchus labrax L. populations. J Fish Biol 2005; 67 : 1746-52.

Castilho R, McAndrew BJ. Population structure of seabass in Portugal: evidence from allozymes. J Fish Biol 1998; 53 : 1038-49.

Chatain B, Saillant E, Peruzzi S. Production of monosex male populations of European seabass, Dicentrarchus labrax L. by use of the synthetic androgen 17[alpha]-methyldehydrotestosterone. Aquaculture $1999 ; 178: 225-34$

Chatain B, Dupont-Nivet M, Haffray P. Le bar, une espèce à fort potentiel pour l'amélioration génétique. Premières Journées Recherche Filière Piscicole, Paris, France, 3-4 juillet 2007.

Chistiakov DA, Hellemans B, Volckaert FAM. Review on the immunology of European seabass Dicentrarchus labrax. Vet Immunol Immunopathol $2007 ; 117: 1-16$.

Colombo L, Barbaro A, Libertini A, Benedetti P, Francescon A, Lombardo I. Artificial fertilization and induction of triploidy and meiogynogenesis in the European seabass, Dicentrarchus labrax L. J Appl Ichthyol 1995; 11: 118-25.

Dupont-Nivet $M$, Vandeputte $M$, Vergnet $A$, Merdy O, Haffray P, Chavanne H, Chatain B. Heritabilities and $G \times E$ interactions for growth in the European seabass (Dicentrarchus labrax L.). Aquaculture $2008 ;(275): 81-7$.

Fauvel C, Suquet M, Dréanno C, Zonno V, Menu B. Cryopreservation of seabass (Dicentrarchus labrax) spermatozoa in experimental and production simulating conditions. Aquat Living Resour 1998; 11 : 387-94.

FEAP. National aquaculture production. http:// www.feap.info/production/countries/default en.asp. 2007.

Felip A, Zanuy S, Carrillo M, Martinez G, Ramos J, Piferrer F. Optimal conditions for the induction of triploidy in the seabass (Dicentrarchus labrax L.). Aquaculture 1997 ; 152 : 287-98

Felip A, Zanuy S, Carrillo M, Piferrer F. Growth and gonadal development in triploid seabass (Dicentrarchus labrax L.) during the first two years of age. Aquaculture 1999; 173 : 389-99.
Fornies MA, Mananos E, Carrillo M, et al. Spawning induction of individual European seabass females (Dicentrarchus labrax) using different GnRHa-delivery systems. Aquaculture $2001 ; 202: 221-34$.

Francescon A, Barbaro A, Bertotto D, et al. Assessment of homozygosity and fertility in meiotic gynogens of the European seabass (Dicentrarchus labrax L.). Aquaculture 2005; 243: 93-102.

Fritsch M, Morizur $Y$, Lambert E, Bonhomme $F$, Guinand B. Assessment of seabass (Dicentrarchus labrax, L.) stock delimitation in the Bay of Biscay and the English Channel based on mark-recapture and genetic data. Fish Res $2007 ; 83: 123-32$.

Garcia De Leon FJ, Canonne M, Quillet E, Bonhomme $F$, Chatain B. The application of microsatellite markers to breeding programmes in the seabass, Dicentrarchus labrax. Aquaculture $1998 ; 159: 303-16$.

Garcia De Leon FJ, Chikhi L, Bonhomme F. Microsatellite polymorphism and population subdivision in natural populations of European seabass Dicentrarchus labrax (Linnaeus, 1758). Mol Ecol 1997 ; 6 : 51-62.

Garcia De Leon FJ, Dallas JF, Chatain B, Canonne M, Versini JJ, Bonhomme F. Development and use of microsatellite markers in seabass, Dicentrarchus labrax (Linneaus, 1758) (Perciforms: Serranidae). Mol Mar Biol Biotechnol 1995; 4 : 62-8.

Gorshkov S, Gorshkova G, Meiri I, Gordin H. Culture performance of different strains and crosses of the European seabass (Dicentrarchus labrax) reared under controlled conditions at Eilat, Israel. J Appl Ichthyol 2004; 20 : 194-203.

Haffray P, Pincent C, Dupont-Nivet M, et al. Heritabilities and $G \times E$ interactions for quality traits in the European seabass (Dicentrarchus labrax L.). Genetics. In aquaculture $I X$ Proceedings of the ninth international symposium on Genetics in aquaculture, Montpellier France, June 26-30, 2006. Aquaculture 2007; (272) (suppl. 1) : S265.

Lemaire C, Allegrucci G, Naciri M, Bahri-Sfar L, Kara H, Bonhomme F. Do discrepancies between microsatellite and allozyme variation reveal differential selection between sea and lagoon in the seabass (Dicentrarchus labrax)? Mol Ecol $2000: 9: 457-67$

Libertini A, Francescon A, Bertotto D, Benedetti $P$, Poltronieri $C$, Barbaro A. Further inves tigations on tetraploidy in the European seabass, Dicentrarchuslabrax (L.). Biol Mar Mediterr 2002; 9: 562-5.

Naciri M, Lemaire C, Borsa P, Bonhomme F. Genetic study of the Atlantic/Mediterranean transition in seabass (Dicentrarchus labrax). $J$ Hered $1999 ; 90: 591-6$.

Patarnello T, Bargelloni L, Calderra $\mathrm{F}$, et al. Mitochondrial DNA sequence variation in the European seabass Dicentrarchus labrax L. (Serranidae) evidence of differential haplotype distribution in natural and farmed population. Mol Mar Biol Biotechnol 1993 ; 2 : 333-7.
Pavlidis M, Koumoundouros G, Sterioti A, Somarakis S, Divanach P, Kentouri M. Evidence of temperature-dependent sex determination in the European seabass (Dicentrarchus labrax L.). J Exp Zool 2000 ; 287 : 225-32.

Peruzzi S, Chatain B. Pressure and cold shock induction of meiotic gynogenesis and triploidy in the European seabass, Dicentrarchus labrax $L$ : relative efficiency of methods and parental variability. Aquaculture 2000 ; 189 : 23-37.

Peruzzi S, Chatain B. Induction of tetraploid gynogenesis in the European seabass (Dicentrarchus labrax). Genetica 2003 ; 119 : 225-8.

Peruzzi S, Chatain B, Saillant E, Haffray P, Menu B, Falguière JC. Production of meiotic gynogenetic and triploid seabass, Dicentrarchus labrax L. 1. Performances, maturation and carcass quality. Aquaculture 2004; 230 : 41-64.

Piferrer F, Blazquez M, Navarro L, Gonzalez A. Genetic, endocrine and environmental components of sex determination and differentiation in the European seabass (Dicentrarchus labrax L.). Gen Comp Endocrinol 2005 ; 142 : 102-10.

Saillant E, Chatain B, Fostier A, Przybyla C, Fauvel C. Parental influence on early development in the European seabass. J Fish Biol $2001 ; 58: 1585-600$

Saillant E, Fostier A, Haffray P, et al. Effects of rearing density, size grading and parental factors on sex-ratios of the seabass (Dicentrarchus labrax L.) in intensive aquaculture. Aquaculture $2003 ; 221$ : 183-206.

Saillant E, Fostier A, Haffray P, Menu B, Thimonier J, Chatain B. Temperature effects and genotype-temperature interactions on sex determination in the European seabass (Dicentrarchus labrax L.). J Exp Zool 2002; 292: 494-505.

Saillant E, Fostier A, Menu B, Haffray P, Chatain B. Sexual growth dimorphism in seabass Dicentrarchus labrax. Aquaculture 2001; 202 : 371-87.

Saillant E, Dupont-Nivet M, Haffray P, Chatain $B$. Estimates of heritability and genotypeenvironment interactions for body weight in seabass (Dicentrarchus labrax L.) raised under communal rearing conditions. Aquaculture 2006 ; 254 : 139-47.

Sansone G, Fabbrocini A, leropoli S, Langellotti AL, Occidente M, Matassino D. Effects of extender composition, cooling rate, and freezing on the motility of seabass (Dicentrarchus labrax L.) spermatozoa after thawing. Cryobiology 2002; 44 : 229-39.

Sola L, De Innocentis S, Rossi AR, et al. Genetic variability and fingerling quality in wild and reared stocks of European seabass, Dicentrarchus labrax. Cah Opt Medit 1998 ; 34 : 273-80.

Vandeputte M, Dupont-Nivet M, Chavanne H, Chatain B. A polygenic hypothesis for sex determination in the European seabass Dicentrarchus labrax. Genetics 2007 ; 176 : 1049-57.

Volckaert FAM, Batargias C, Canario A, et al. The European seabass (Dicentrarchus labrax L.) and its genomic resources. In : Kocher TD, Kole C, eds. Genome Mapping and Genomics. Fishes and Aquatic Animal, Vol. 2. Berlin; Heidelberg: Springer-Verlag, 2008. 